\title{
Filigrane
}

Écoutes psychothérapiques

\section{La petite fille et l'éléphant. Violences inconcevables, transfert et mise en récit}

\section{Louise Grenier}

Volume 19, numéro 1, printemps 2010

Adieu Oedipe, bonjour Narcisse ?

URI : https://id.erudit.org/iderudit/044180ar

DOI : https://doi.org/10.7202/044180ar

Aller au sommaire du numéro

Éditeur(s)

Revue Santé mentale au Québec

ISSN

1192-1412 (imprimé)

1911-4656 (numérique)

Découvrir la revue

Citer cet article

Grenier, L. (2010). La petite fille et l'éléphant. Violences inconcevables, transfert et mise en récit. Filigrane, 19(1), 67-75.

https://doi.org/10.7202/044180ar
Résumé de l'article

Comment suppléer aux déchirures de la mémoire ? Comment raconter une violence extrême - physique ou psychologique - vécue dans un temps qui précède la parole, et alors qu'aucune possibilité de représentation et de récit n'existe ? Dans La petite fille et l'éléphant, j'émets l'hypothèse que la violence contre soi constitue une défense contre un état d'abandon psychique. La cure analytique pourrait être ce lieu qui permet à la fois la symbolisation de cet état d'abandon et la mise en récit de soi dans son rapport à des violences primitives. Partant de mes souvenirs et expériences cliniques, des témoignages apportés par des romanciers, philosophes et collègues psychanalystes, j'interroge ce que j'appelle « l'expérience de l'inconcevable » et propose une approche qui favorise la mise en récit de soi (qui inclut les silences de son passé) à partir du transfert. 


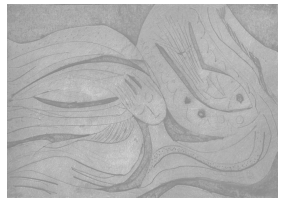

\title{
La petite fille et I'éléphant. Violences inconcevables, transfert et mise en récit ${ }^{1}$
}

\section{Louise Grenier}

\begin{abstract}
Comment suppléer aux déchirures de la mémoire? Comment raconter une violence extrême - physique ou psychologique - vécue dans un temps qui précède la parole, et alors qu'aucune possibilité de représentation et de récit n'existe? Dans La petite fille et l'éléphant, j'émets l'hypothèse que la violence contre soi constitue une défense contre un état $d$ 'abandon psychique. La cure analytique pourrait être ce lieu qui permet à la fois la symbolisation de cet état d'abandon et la mise en récit de soi dans son rapport à des violences primitives. Partant de mes souvenirs et expériences cliniques, des témoignages apportés par des romanciers, philosophes et collègues psychanalystes, j'interroge ce que j'appelle "l'expérience de l'inconcevable» et propose une approche qui favorise la mise en récit de soi (qui inclut les silences de son passé) à partir du transfert.
\end{abstract}

Le désert croît, malheur à qui recèle des déserts! (Nietzche, 1971, 366)

\begin{abstract}
Creteris et énoncé de Nietzsche (1844-1900) s'est imposé à mon esprit lorsque je préparais cet article. Je l'ai donc suivi comme un panneau indicateur sur les routes de ma mémoire. J'ai ouvert mon vieil exemplaire de Ainsi parlait Zarathoustra et voici ce que j'ai trouvé sur la page de garde: "Louise Grenier, Dakar, 6 janvier 1975, p. 136 ». L'inscription gardait la trace d'une de mes flâneries dans la capitale du Sénégal. D’un de ces mardis où je disposais de quelques heures avant d'aller à l'Hôpital psychiatrique de Fann. En passant par la Place de l'Indépendance, j'avais dû m'arrêter chez Clairafrique, une librairie dont j'ai retrouvé le nom et l'adresse grâce à Google. Mais pourquoi avais-je également noté la page 136? Serait-ce une autre indication menant au refoulé, l'adresse d'un souvenir oublié de cette époque? Trentetrois ans plus tard, à la page 136, je relis ce passage poétique de Nietzsche:
\end{abstract}

Il est en moi quelque chose d'inassouvi, d'inassouvissable, et qui plus haut prétend parler. Il est en moi désir d'amour, qui parle lui-même langage d'amour. [...] Lumière suis; ah! que ne fussé-je nuit! Mais c'est ma solitude que de lumière je sois ceint.

Ah! ne fussé-je obscur et nocturne ! Comme aux seins de la lumière alors je voudrais boire mon lait! (Nietzche, 1971, 136) 
L'écrivain comble les lacunes de nos propres symbolisations, il met en mot le réel, et pas seulement le réel du monde interne et externe, mais ce qu'il y a d'inconcevable et d'inaccessible à la remémoration. Nietzsche a mené une vie solitaire, et de son propre aveu, sans amante. Son biographe, Daniel Halévy (1944), raconte qu'au cours d'une de ses dernières promenades, le philosophe poète avait été happé par le regard d'une petite fille, regard qui dans un instant d'éternité avait semblé l'étreindre. Ce contact fugitif avec l'autre ne lui avait-il pas renvoyé le reflet perdu de l'enfant qu'il avait été, ou qu'il était encore?

"Le désert croît», écrit Nietzsche. Et il ajoute cette annotation manuscrite: "Malheur à celui qui devient désert. Désert est la faim qui creuse jusqu'au cadavre [...] Sable est la mère qui a mâché son enfant avec un poignard volant dans la peau $^{2}$.» Désert qui associe la faim dévorante de la mère au devenir de l'enfant. Désert qui identifie l'enfant dans le sillage des violences de la mère. Un désert au revers du désir en quelque sorte... Telle est la vision nietzschéenne du malheur, un désert intérieur qui s'étale autour d'une absence. Sans doute parce que l'un des destins psychiques des violences inconcevables - qu'elles proviennent de soi, d'un autre ou de l'environnement - est la désertification de l'être. Désertification qui garde la trace en creux de la disparition du sujet comme être de désir. Tout être maltraité, négligé ou violenté recèle des déserts par lui ignorés. Pour survivre, il s'est identifié à son agresseur, a incorporé le mal qui le tue, et dans cette identification imaginaire, a disparu en même temps que ses objets d'amour.

Après Mélanie Klein, Jacques Lacan $(1975,119)^{3}$ a posé l'existence d'un surmoi primitif distinct de l'instance interdictrice décrite par Freud lors de l'Edipe. Alors que cette dernière borne la satisfaction pulsionnelle, le surmoi tyrannique ignore toute limite. Produit de l'incorporation d'une figure de toute-puissance destructrice, il échappe à la loi paternelle. Ce type de surmoi non soumis à loi symbolique se constitue à partir des expériences traumatiques subies par l'enfant. Ces traumatismes précoces sont forclos ${ }^{4}$ faute de pouvoir être élaborés dans un système symbolique sous l'égide de la loi paternelle. Rejetés dans le réel, ils feront retour via des pensées et conduites autodestructrices: haine de soi, anorexie, troubles somatiques, délires mélancoliques et sentiment d'une perte irréparable.

Les violences de l'Autre - individu ou environnement - s'étaient sur des carences affectives et narcissiques, ou provoquent l'effondrement psychique de la figure maternelle. Ceux qui ont vécu la faim et la terreur, le mépris et la torture, l'exclusion et les Camps de la mort, témoignent d'un effet de sidération dans un contexte de solitude absolue.

Mon hypothèse de départ est que la mise en mots d'un vécu, d'un réel forclos de la représentation (symbolique), est la condition de possibilité de la mise en récit de soi. Mise en récit qui à son tour permet à son auteur de parcourir son passé et de consentir à son histoire. Les littéraires distinguent la fictionnalisation de l'historicisation, la vérité narrative de la vérité historique. Le récit historique est associé au témoignage alors que la fictionnalisation «implique un surplus imaginaire» (Roy). Le récit de soi ne rend pas compte de la réalité objective mais de la réalité psychique 
au moment de l'écriture. Il s'agit, comme l'écrivait Virginia Woolf dans Instants de vie (Woolf, 1988), de décrire la personne à qui les choses arrivent, et non ce qui arrive.

Voilà qui soulève d'autres questions : qu'en est-il d'événements qui remontent à la préhistoire du sujet? Comment raconter des expériences survenues avant l'entrée dans la parole et ne se manifestant qu'à travers la répétition et le transfert? L'image de La petite fille et de l'éléphant illustre magnifiquement la problématique de l'immensité de la mémoire perdue.

\section{La petite fille et l'éléphant}

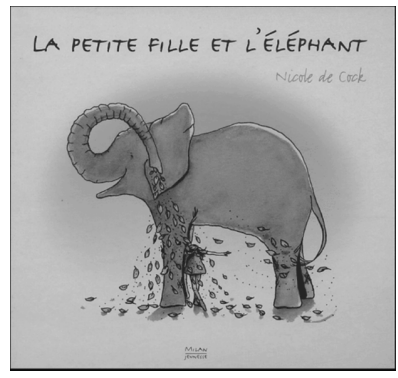

Comme le désir sexuel, la mémoire ne s'arrête jamais. Elle apparie les morts aux vivants, les être réels aux imaginaires, le rêve à l'histoire. (Ernaux, 2008).

L'album intitulé La petite fille et l'éléphant (de Cock, 2005) raconte l'amour d'une petite fille pour un éléphant rencontré au zoo, et qui un jour retourne en Afrique. S'ensuit une recherche intense pour le retrouver, "mais l'Afrique est si vaste...» L'éléphant symbolise à la fois l'immensité de la perte et la mémoire de l'objet. Dans l'image ci-dessus, des fragments tombent de la trompe de l'éléphant et se déversent sur et autour de l'enfant. Que sont ces fragments? Des souvenirs, des rêves nourriciers qui rappellent l'objet perdu, et avec lui le désir que tout soit comme avant? Ce qui frappe ici est la disproportion des forces en présence entre d'une part, la petite fille et son objet d'amour, entre son désir de le retrouver et l'impossibilité de le reprendre en soi. Comme certaines expériences, de celles qui font violence au sujet, qui semblent à tout jamais exclues de la mémoire, et qui paraissent inutilisables pour la narration de son histoire et la fabrication de son identité. Par des voies énigmatiques, ces expériences rejoignent une matière pulsionnelle primitive qui nourrit la répétition autodestructrice. Or, celle-ci ne pourra cesser que si nous parvenons à remplacer la répétition par le découvrement de ce qu'elle dérobe au discours. La pratique de l'analyse n'est-elle pas une façon d'étreindre la mémoire afin d'y ressaisir les souvenirs de l'objet perdu, et soi-même avec lui?

\section{De l'archaïque à la mise en récit: Saxa loquuntur (les pierres parlent) (Freud, 1895, 84)}

Derrida (1996), s'appuyant sur l'article « Notes sur le "bloc-notes magique" » (Freud, 1925, 121-124), souligne que Freud conçoit l'appareil psychique comme une 
machine à écrire qui produit de façon permanente la scène de l'écriture (Freud, 1973, 153-160). Le vécu infantile (au sens de réel lacanien) laisse des traces ineffaçables qui, bien qu'illisibles, ne cessent de pousser vers le conscient. Non récupérables comme textes, elles déterminent - et parfois bloquent - les voies d'accès au désir et au lien affectif. Ces traces mnésiques réapparaissent dans le transfert passionnel et des liens destructeurs. Ainsi, le sujet maintient le lien à l'Autre de la violence (ou à son tenant-lieu) et tous nos efforts interprétatifs pour l'en séparer s'avèrent inopérants. Pourquoi? Pourquoi est-il si important de maintenir le lien à l'autre destructeur, abuseur ou absent? Que signifie cette captation? À quel désir croit-il satisfaire en se faisant objet du désir d'un autre, surtout si ce désir implique de s'auto-sacrifier?

Pour certains analysants, s'engager en analyse, c'est tenter de regagner un savoir perdu avec le désir (Balmary, 1986), et pour ce qui concerne mon propos, un savoir portant sur le désir de l'Autre. L'Autre en tant qu'altérité incorporée, et que l'analyste incarne dans le transfert. Notre fonction ne s'arrête pas là : elle consiste d'une part, à rechercher les indices d'un inconnu à interpréter ou à reconstruire, d'autre part, à recueillir des affects à la limite du supportable : impuissance, terreurs, désirs de mort (Bion, 1970, 83-101). Il s'agit d'ouvrir un espace d'identification primaire avec une altérité que l'analyste ne conçoit pas encore. C'est ce que je veux dire par « une écoute de l'inconcevable». L'écoute de l'analyste sépare le sujet de son agresseur intérieur un surmoi tyrannique et cruel - afin de laisser émerger de sous la glace un enfant laissé pour mort.

«Revenant d'une absence mortelle», comment raconter le néant? Le survivant doit lutter contre la tentation de l'effacement des traces - cet enfant mort qu'il porte en lui - et contre la surdité de son entourage. Il arrive qu'il veuille se faire disparâ̂tre totalement: «Je ne voudrais que l'oubli, rien d'autre» (Semprun, 1994, 211). Il croit que son histoire est incommunicable. Il se trompe, pense Jorge Semprun:

On peut toujours tout dire, l'ineffable dont on nous rebattra les oreilles n'est qu'alibi. [...] On peut toujours tout dire, le langage contient tout. On peut dire l'amour le plus fou, la plus terrible cruauté.. [...] On peut tout dire de cette expérience. Il suffit d'y penser. Et de s'y mettre. D'avoir le temps, sans doute, et le courage, d'un récit illimité, probablement interminable, illuminé [...] quitte à n'être plus que le langage de cette mort, à vivre à ses dépens, mortellement. [...]

Mais peut-on tout entendre, tout imaginer? En auront-ils la patience, la passion, la compassion, la rigueur nécessaire? Le doute me vient, dès ce premier instant, cette première rencontre avec des hommes d'avant, du dehors [...] Je me suis vu dans leur œil horrifié pour la première fois depuis deux ans. [...] Car je n'avais pas vraiment survécu à la mort, je ne l'avais pas évitée. [...] J'étais un revenant en somme. Cela fait toujours peur les revenants. (Semprun, 1994, 26-27)

Il me fallait citer ce passage longuement pour montrer que dans les cas extrêmes, la victime est indissociable de son agresseur et qu'elle porte un mort en elle. Dans l'imaginaire, elle est piégée dans une fusion mortifère, autodestructrice. La violence 
subie ou agie, celle qui déshumanise, entraîne une fissure dans le moi, un clivage narcissique entre une partie qui sait mais ne souffre pas, et une partie qui souffre mais ne sait pas. Il s'agit pour l'analyste de permettre la rencontre entre ces deux parties, ce qui n'est guère aisé. Une citation de Kertész éclairera mon propos:

Il s'avéra que je n'écrivais pas pour chercher du plaisir, au contraire, il s'avéra qu'en écrivant je cherchais la souffrance la plus aiguë possible, à la limite du supportable, vraisemblablement parce que la souffrance est la vérité, quant à savoir ce qu'est la vérité, écrivis-je, la réponse est simple : la vérité est ce qui me consume [...]. (Kertész, $1995,101)$

\section{L'absence traumatique de l'autre}

La plus grande désolation est l'absence de l'autre, un proche qui se détourne quand vous abordez le récit d'une souffrance sans nom. Le survivant éprouve le besoin impérieux de dire son histoire pour se délivrer des fantômes de son passé, mais le problème dans ce cas n'est pas tant de pouvoir parler que d'être écouté, de trouver un autre qui ne se dérobe pas. «Le récit non écouté est un traumatisme aussi grave que l'épreuve initiale» (Cojean, 1995), ce que confirment les cauchemars récurrents des revenants de la Shoah et de bien d'autres victimes de situations limites.

Cette surdité de l'autre explique le silence dans lequel se sont repliés tant de rescapés de leur propre enfance. Ce qu'ils craignent plus que tout: n'être ni écoutés ni crus par leurs proches. Cette «douleur à l'état pur» (Levi, 1987, 90), qui n'est apaisée par rien ni personne, est étrangement pire que l'horreur quotidienne du Camp de la mort. Primo Levi la compare à l'effet produit par l'indifférence des autres à la désolation du petit enfant abandonné: "Alors une désolation totale m'envahit, comme certains désespoirs enfouis dans les souvenirs de la petite enfance [...] 》 (Levi, 1987, 90). L'autre s'absente, ne veut pas entendre, et cette absence désespère le survivant. Personne ne fera écho à cette part de lui enchaînée à son passé. Il habite une région qui va bien au-delà du trauma : celle de l'inconcevable.

Dans la cure de patients ayant survécu à des violences extrêmes, nous sommes placés face à de multiples contradictions : reconnaître la cruauté d'un événement qui fait effraction dans la psyché, témoigner de l'inconcevable d'une expérience terrifiante; revenir sur les lieux d'une absence. Dans le transfert, l'analyste fait fonction de témoin tiers, de témoin garant de la parole et de la mémoire inconsciente tout au long de la cure. Comme Chiantaretto (2005), je pense que l'analyste est aussi un historien de l'infantile qui associe ses constructions au témoignage de l'analysant.

Les témoins survivants nous disent quelque chose de la possibilité, plus ou moins présente pour chacun au début de son existence, «d'une défaillance mortelle de l'environnement [...] et quelque chose de la dépendance vitale à la psyché de l'autre, du besoin psychique d'un témoin pour exister» (Chiantaretto, 2005, 140). C'est un savoir qui ne se laisse pas oublier, et qui devient une vérité plus vraie que la vie «réelle». Or, cette vérité est indissociable de l'abandon, comme l'écrit Jean Améry: 
En dernier ressort l'expérience de la persécution était celle d'une extrême solitude. Ce qui m'importe, c'est d'être délivré de cet état d'abandon qui persiste toujours. (Améry, 1995, 153)

L'expérience de ceux qui ont survécu aux camps de la mort nazis et à la torture est incomparable. Il semble que rien ne peut en approcher l'horreur, sauf peut-être la souffrance infligée à des enfants sans défense par des adultes indifférents ou cruels. Ces enfants traversent un enfer et ils ne peuvent pas vous le dire, ni plus tard vous le raconter parce que les événements ont eu lieu dans leur préhistoire et en secret. Leur premier contact avec la vie est marqué par des abus ou des carences. L'Autre dont il dépend absolument est la cause d'une douleur infinie et incommunicable.

Parmi les victimes de violence, il y a donc ceux qui peuvent raconter - ils sont sujets d'un récit, et ceux qui ne peuvent pas raconter - ils sont tout au plus objets de discours. La question du rapport entre les récits des survivants des camps de la mort et l'absence de récit de ceux qui ont traversé des agonies primitives m'est venue pendant la lecture de Si c'est un homme de Primo Levi. Lévi témoigne dans l'immédiat après-coup de son expérience concentrationnaire d'Auschwitz où il a été interné de février 1944 à janvier 1945.

Son témoignage rejoint par certains traits des vécus archaïques de violence physique et psychologique : abandon, rejet et abus de toutes sortes. Dans tous les cas, ces violences provoquent un sentiment de désolation absolue chez la victime. C'est cela, le fond de l'abîme. De ne pas exister, de n'être plus que l'objet de la violence - ou de la jouissance - de l'Autre. L'Autre, ou un de ses représentants, devient pour vous la seule réalité, or, c'est une réalité qui vous exclut, qui vous tue.

Pour survivre, le sujet retranche la part de soi qui contient la mémoire de l'événement destructeur et sa douleur. Il peut arriver aussi qu'il s'absente de lui-même, qu'il ne ressente plus rien. Le fait peut être connu, le récit en est possible, mais de l'extérieur. C'est un récit sans sujet, un récit désaffecté par celui-là même qui en est le personnage principal et le narrateur. Comme s'il n'y avait personne au-dedans de soi.

On peut retrouver chez certains analysants des états de disparition subjective et d'abandon ${ }^{5}$ qui évoquent l'expérience concentrationnaire, à la différence qu'ils en ignorent la cause et ne peuvent en témoigner, sinon à travers leurs symptômes névrotiques ou psychotiques, leurs cauchemars et leurs malheurs. Comme l'adolescent d'Être sans destin de Kertész, ils ont l'impression d'être tombés soudain au beau milieu d'une pièce de théâtre insensée dans un rôle qu'ils ne connaissent pas. Impossible de trouver un sens aux faits et gestes des gens qui les entourent, bourreaux et victimes. Tous sont prisonniers d'un dispositif destiné à les broyer.

Jean Améry a été torturé dans le but de lui arracher des renseignements. C’est «l'événement le plus effroyable qu'un homme puisse garder au fond de soi » (Améry, 1995, 61). Le premier coup fait comprendre au détenu qu'il est sans défense, et que ce geste renferme tout ce qui va suivre à l'état embryonnaire. On a le droit de le frapper. On peut faire de lui tout ce qu'on voudra. À l'extérieur, personne n'en saura rien, 
et personne ne viendra à son secours : «Finalement le viol physique par l'autre se mue en acte d'anéantissement existentiel dès lors qu'il n'y a plus d'aide à espérer» (Améry, 1995, 73), écrit-il. Et il ajoute:

L'attente d'une aide extérieure est un élément constituant du psychisme au même titre que la lutte pour la vie. Je viens tout de suite, dit la mère à l'enfant qui gémit de douleur, tu vas avoir un biberon chaud, tu vas avoir une tasse de thé, on ne pas te laisser souffrir comme ça! Je vous prescris un médicament, tranquillise le médecin, cela vous aidera à surmonter le mal. [...] Dans presque toutes les situations de la vie la blessure physique va de pair avec l'attente d'une aide extérieure: la première est compensée par la seconde. (Améry, 1995, 73)

Cette expérience est incommunicable. La torture a aussi un caractère indélébile: "Celui qui a été torturé reste torturé », écrit Jean Améry (1995, 83). Pour faire comprendre à autrui ce que fut sa douleur, il faudrait l'infliger à l'autre, il faudrait se changer soi-même en tortionnaire. Ce que d'une certaine façon, il concrétisera en se suicidant. Primo Levi insiste sur le sentiment de culpabilité et la mémoire de l'offense du survivant qui tenaillent le survivant:

Le souvenir d'un traumatisme, souffert ou infligé, est lui-même traumatisant parce que son rappel fait souffrir ou, pour le moins perturbe; celui qui a été blessé a tendance à refouler le souvenir pour ne pas renouveler la douleur; celui qui a été blessé, repousse le souvenir dans les profondeurs afin de s'en libérer, d'alléger son sentiment de culpabilité. (Levi, 1989, 24)

L'offense est inguérissable: le surmoi primitif en conserve le souvenir en continuant d'infliger de la souffrance psychique. L'offensé devient cette image odieuse reflétée par les miroirs meurtriers. C'est un Narcisse défait et ravagé qui reste figé, non dans la contemplation de son image idéale, mais dans la quête angoissée de ce qu'il a perdu. Dans ce retour forcé au stade du miroir, il ne trouve que l'écho d'un ratage. D'où l'importance pour le thérapeute de réparer la blessure faite à l'altérité. Comment?

\section{L'analyste, un historien de l'infantile}

Dans la cure des personnes violentées ou qui s'auto-violentent, il ne s'agit pas seulement de raconter les violences de l'Autre, traumatiques ou non, mais de remonter jusqu'aux états d'abandon du moi. De dévoiler la perte d'objet maternel protecteur sous-jacente à l'expérience de violence. J'irais même plus loin, le sujet peut survivre à toutes sortes d'épreuves, en revenir, à condition d'avoir maintenu un lien affectif avec un autre (Antelme, 1957). Ce qui est vraiment traumatique, ce n'est pas l'événement en soi, nonobstant sa puissance dévastatrice, mais le fait que le sujet soit précipité dans une solitude absolue et mortelle. D'où un fond d'angoisse de mort difficilement repérable dans la séance mais toujours agissante dans le transfert. L'analyse elle-même devient pour le sujet une question de vie ou de mort. La figure 
de l'analyste se dédouble en fonction des projections du sujet: tantôt cruelle, tantôt secourable, le sujet y faisant lecture des traits gravés sur le tableau de cire de l'inconscient, traits dont il tente de reconstituer le texte premier.

$\mathrm{Au}$ fil des séances et du transfert, le thérapeute découvre chez son patient un «savoir» portant sur le désir de l'Autre. Savoir délirant bien sûr, mais qui le tient captif du désir d'un autre auquel il ne cesse de se sacrifier - c'est la capture sacrificielle dont parle Lacan (1971), capture qu'il associe au masochisme. Le masochisme consiste ici non à souffrir mais à se faire objet du désir de l'Autre, objet de sa jouissance, de sa cruauté.

Dans l'écoute il nous arrive de «souffrir la peine de l'autre » écrivent Davoine et Gaudillière (2006, 117-118, 199). D'être cet autre horrifié appelé à témoigner d'un état du moi souffrant et dissocié. Loin d'affecter notre objectivité, c'est là un seuil à franchir pour y accéder. L'analyste en plus d'enregistrer ce qu'il ressent, doit trouver le moyen d'en faire état en séance. Autrement dit, ce qui est retranché du sujet reparaît chez l'analyste qui peut dès lors le restituer au patient. Nous dirons alors que nous sentons, imaginons, rêvons à la place du patient.

L'analyste n'est pas seulement le spectateur d'un scénario désespéré rejoué dans la cure, il n'est pas seulement l'auditeur d'un récit raconté et enfin écouté, il est aussi le représentant du monde symbolique qui à ce titre a fonction de mettre fin aux violences de l'Autre. C'est en tiers témoin qu'il reconnaît les fantômes du passé pour les identifier et permettre à son analysant de s'en séparer. L'écoute doit être ici l'expression d'une présence qui fait contrepoids aux silences - et aux absences - du proche secourable. Une écoute qui permet l'historicisation du sujet, ce que Freud appelle reconstruction, et que nous pourrions appeler également «fiction». La fiction étant ce qui restitue au sujet une part de son passé, remanié certes, repensé et marqué au coin des pulsions de vie?

Louise Grenier 810, av. Champagneur, app. 225

Outremont, Qc

$\mathrm{H} 2 \mathrm{~V} 4 \mathrm{~S} 3$

Courriel : grenier.louise@uqam.ca

\section{Notes}

1. Ce texte est la version remaniée d'une conférence que j'ai prononcée pour le Département de psychiatrie de l'Hôpital Maisonneuve-Rosemont, à l'invitation du Dr Gilles Chagnon, responsable de la formation continue.

2. Note manuscrite: 2.115.9. Les filles du désert sont des prostituées.

3. «Le surmoi finit par s'identifier à ce qu'il y a de plus ravageant, de plus fascinant, dans les expériences primitives du sujet. Il finit par s'identifier à ce que j'appelle la figure féroce, aux figures que nous pouvons lier aux traumatismes primitifs, quels qu'ils soient, que l'enfant a subis.» Voir aussi: Anne Juranville $(1993,92)$. Dans la psychose, le caractère mortel du surmoi est l'effet, inversement, du rejet de la parole symbolique.

4. Le terme de forclusion est au centre de la théorie lacanienne des psychoses. Il marque le rejet du 
signifiant fondamental, pierre angulaire sur laquelle se construit l'appareil psychique du sujet non psychotique. Le père en tant que symbole, «le Nom-du-Père», constitue ce signifiant fondamental qui permet l'accès au stade symbolique. La mère exerce un rôle privilégié dans la transmission à son enfant de ce premier symbole qu'est la fonction paternelle.

5. Il ne s'agit pas ici de sentiment d'abandon ou d'expériences d'abandon, mais d'un état figé du moi, sorte de désert intime qui va grandissant.

\section{Références}

AMÉRY, J., 1966/1995, Par-delà le crime et le châtiment, Essai pour surmonter l'insurmontable, Acte Sud, Babel.

ANTELME, R., 1957/2005, L'espèce humaine, Gallimard, Paris.

BALMARY, M., 1986, Le sacrifice interdit, Le sacrifice interdit. Freud et la Bible, Grasset, Paris.

BION, 1970, L'attention et l'interprétation, in L'opacité de la mémoire et du désir, Payot, Paris.

CHIANTARETTO, J.-F., 2005, Le témoin interne. Trouver en soi la force de résister, Aubier/La psychanalyse prise au mot, Paris.

COCK, N. de, 2005, La petite fille et l'éléphant. http://livre.fnac.com/a1603914/Nicole-de-Cock-La-petitefille-et-l-elephant

COJEAN, A., 1995, Les mémoires de la Shoah in Le Monde (29 avril). http://www.antirev.org/textes/Cojean95a/index.html

DAVOINE, F., MAX GAUDILLIÈRE, J.-M., 2006, Histoire et trauma. La folie des guerres, Stock, Paris.

DERRIDA, J., 1996, Mal d'archives, Galilée, Paris.

ERNAUX, A., 2008, Les années, Gallimard, Paris.

FREUD, S., 1895/1990, Étiologie de l'hystérie, in Névrose, psychose et perversion, PUF, Paris.

FREUD, S., 1925/1985, Notes sur le «bloc-notes magique» in Résultats, idées, problèmes, Tome 2, PUF, Paris.

FREUD, S., 1973, La naissance de la psychanalyse, PUF, Paris.

HALEVY, D., 1944/1977, Nietzsche, Grasset/Pluriel, Paris.

JURANVILLE, A., 1993, La femme et la mélancolie, PUF/écriture, Paris.

KERTÉSZ, I., 1995/2003, Kaddish pour l'enfant qui ne naîtra pas, Actes Sud, Paris.

LACAN, J., 1975, Les Écrits techniques de Freud, Seuil, Paris.

LACAN, J., 1971, Séminaire Livre XI, Les quatre concepts fondamentaux de la psychanalyse, Seuil, Paris.

LEVI, P., 1989, Naufragés et rescapés, Gallimard, Paris.

LEVI, P., 1987/2004, Si c'est un homme, Julliard, Paris.

NIETZSCHE, F., 1971, Ainsi parlait Zarathoustra, Idées/Gallimard, Paris.

ROY, L., Fictionnalisation et historicisation ou le paradoxe de la violence (im)matérielle; http://figuresdeviolence.org/fictionnalisation-et-historicisation-ou-le-paradoxe-de-la-violence-immat-rielle

SEMPRUN, J., 1994, L'écriture ou la vie, Folio/Gallimard, Paris.

WOOLF, V., 1988, Instants de vie, Biblio/Stock, Paris. 